\title{
VIABILIDAD TÉCNICO AMBIENTAL DE LA DISPOSICIÓN FINAL DEL LODO GALVÁNICO EN LA FABRICACIÓN DE LADRILLOS REFRACTARIOS
}

\section{ENVIRONMENTAL TECHNICAL FEASIBILITY OF THE FINAL DISPOSAL OF GALVANIC SLUDGE IN THE MANUFACTURE OF REFRACTORY BRICKS}

(iD) Héctor Eduardo Sánchez Vargas ${ }^{1}$

'Universidad de Camagüey, Camagüey, Cuba.

Correspondencia:

Dr. Héctor Eduardo Sánchez Vargas.

hector.sanchez@reduc.edu.cu

\section{RESUMEN}

Cuidar el medio ambiente y evitar los efectos que producen los contaminantes y desechos peligrosos promovieron como objetivo de este trabajo evaluar la viabilidad técnica y ambiental de la disposición final del lodo galvánico en la fabricación de ladrillos refractarios. Entre los materiales y métodos se aplicaron ensayos a muestras de lodo galvánico aplicando la espectrometría de absorción atómica; la alternativa se seleccionó considerando las buenas prácticas y la cercanía fuente generadora - destino final; desde lo ambiental, ésta se evaluó con una prueba de lixiviación en el marco de un diseño experimental determinando cromo hexavalente $\left(\mathrm{Cr}^{6+}\right)$. Técnicamente la evaluación partió del diseño tecnológico para la aplicación de la alternativa de disposición del lodo. Se midió el cumplimiento de los requisitos de calidad del ladrillo refractario: resistencia de la compresión y porciento de absorción de agua. Para cada una de estas variables se ajustaron modelos matemáticos empleando la herramienta Curve Fitting del software MATLAB. Se concluyó que es viable técnica y ambientalmente la disposición final de lodo galvánico en la mezcla para la fabricación de ladrillos refractarios; los ladrillos fabricados con las concentraciones de lodo determinadas, cumplirán con los requisitos de calidad exigidos por la empresa; el modelo matemático obtenido demostró la capacidad para determinar la resistencia a la compresión en función de la concentración de lodo y la temperatura de cocción; con una concentración de hasta el $4 \%$ se garantiza que el ladrillo sea seguro y que durante su cocción no se generen gases con presencia de $\mathrm{Cr}^{6+}$.

Palabras clave: lodo galvánico, disposición de sólidos, metales pesados, desechos peligrosos

\section{ABSTRACT}

Caring for the environment and avoiding the effects produced by pollutants and hazardous wastes promoted as the objective of this work to evaluate the technical and environmental feasibility of the final disposal of galvanic sludge in the manufacture of refractory bricks. Among the materials and methods, the following were applied tests 
on galvanic sludge samples applying atomic absorption spectrometry; the alternative was selected considering good practices and the proximity of the generating source - final destination; From the environmental point of view, it was evaluated with a leaching test within the framework of an experimental design determining hexavalent chromium $\left(\mathrm{Cr}^{6+}\right)$. Technically, the evaluation started from the technological design for the application of the sludge disposal alternative. Compliance with the quality requirements of the refractory brick was measured: compression resistance and percentage of water absorption. Mathematical models were adjusted for each of these variables using the Curve Fitting tool of the MATLAB software. It was concluded that the final disposal of galvanic sludge in the mixture for the manufacture of refractory bricks is technically and environmentally viable; the bricks manufactured with the determined sludge concentrations will meet the quality requirements demanded by the company; the mathematical model obtained demonstrated the ability to determine the compressive strength as a function of the sludge concentration and the firing temperature; with a concentration of up to $4 \%$, it is guaranteed that the brick is safe and that during its firing, gases with the presence of $\mathrm{Cr}^{6+}$ are not generated.

Key words: galvanic sludge, solid disposal, heavy metals, hazardous waste

\section{INTRODUCCIÓN}

La presencia de contaminantes en los residuos generados por la actividad humana amenaza el ambiente y la salud de todas las especies vivientes, algunos no causan efectos significativos sobre el medio que nos rodea, pero otros provocan gran afectación desde el punto de vista ecológico.

Los lodos galvánicos, generados por empresas metalmecánicas, son considerados desechos peligrosos en gran parte del mundo (Agencia Presidencial de Cooperación, 2017; Andreola y col., 2006; CITMA, 1999; Organisation for Economic Cooperation and Development (OECD), 2009; Presidencia de la República, 2005; Scottish Environment Protection Agency (SEPA), 2021; UNEP, 2000). Este residuo industrial contiene cantidades variables de metales pesados, los que dependiendo de su dinámica pueden acumularse en el suelo y generar efectos adversos, ser absorbidos por plantas que posteriormente son de consumo humano o animal y alcanzar el manto freático. En algunos suelos y resevorios acuíferos ya se han identificado estos elementos como parte de sus principales contaminantes.

Existen diferentes tipos de tratamientos a residuales líquidos, que si bien, en algunos casos llegan a solucionar problemas de contaminación de las aguas, generan grandes cantidades de lodo rico en metales pesados como hierro, cinc, cromo trivalente, níquel, entre otros. Estos residuos, si no son dispuestos en lugares seguros, pueden llegar a convertirse en potenciales agentes contaminantes.

Es conocido que dentro de los metales pesados existen algunos que desempeñan roles vitales en los organismos vivientes cuando están a niveles de trazas (conocidos como micro elementos). Cuando su concentración en el organismo supera la necesaria o no le alcanza, se presentan graves trastornos. Entre las principales enfermedades asociadas a la intoxicación con metales pesados están las relacionadas con alteraciones al metabolismo y los daños hepáticos y renales. También pueden producir daños neurológicos, hematológicos, inmunológicos y cardiovasculares (Becerra, Soria, Jaramillo, y Moreno, 2014; Rodríguez, 2017; Tirado, González, Martínez, Wilches, y Celedón, 2015). Los metales pesados incluidos en los lodos galvánicos que mayor peligrosidad poseen son el cromo, plomo, cadmio, arsénico y mercurio. El cromo en estado trivalente es un nutriente esencial para los humanos y la falta de este puede afectar el corazón y ocasionar trastornos metabólicos y diabetes, pero la toma en exceso también tiene sus efectos sobre la salud, como las erupciones cutáneas. El $\mathrm{Cr}^{6+}$ representa un peligro para la salud de los humanos, provocando reacciones alérgicas y erupciones cutáneas, además de irritación en 
la nariz y sangrado después de ser respirado. También ocasiona debilitamiento del sistema inmune, daño en los riñones e hígado, problemas respiratorios, alteración del material genético, malestar de estómago, úlceras y cáncer (Rodríguez, 2017).

El Ministerio de Ciencia, Tecnología y Medio Ambiente de Cuba (CITMA), ha identificado como los principales problemas ambientales del país a la degradación de los suelos, las afectaciones a la cobertura forestal, la contaminación, la pérdida de diversidad biológica y la carencia de agua (Somoza Cabrera, 2009). Este organismo ha definido dentro de los diferentes programas ambientales encaminados a la solución o mitigación de las afectaciones que se producen al medio ambiente, programas para la reducción de la contaminación que comprenden y conjugan conjuntos de mecanismos e instrumentos de gestión ambiental, orientados a prevenir, reducir y controlar la contaminación proveniente de las principales fuentes contaminantes identificadas en el ámbito nacional.

Paralelamente se han ejecutado acciones encaminadas a la rehabilitación y mantenimiento de los sistemas de tratamiento existentes, la remodelación tecnológica, la reorientación de prácticas encaminadas al aprovechamiento de residuales y programas de producción más limpias, los cuales por su definición, traen consigo la aplicación continua de una estrategia ambiental preventiva integrada a los procesos, producciones y servicios, para incrementar su eficiencia, reducir los riesgos para los seres humanos, el ambiente y lograr la sostenibilidad del desarrollo económico.

La empresa mecánica objeto de estudio genera una cantidad apreciable de lodo galvánico que en la actualidad es depositado en un vertedero aprobado para tal fin por el CITMA, pero que, a pesar de su disposición en espacio confinado, los grandes volúmenes y la acumulación durante años ha creado un problema ambiental de importancia. Ante esta situación, se ha planteado la necesidad de disponer de forma segura del lodo galvánico generado en la empresa, sin que se afecte el medio ambiente. Por las características tecnológicas de esta empresa y el tipo de producciones estos lodos son especialmente ricos en hierro y cromo, siendo este último el de mayor peligrosidad.

Para darle solución a este tipo de problemas se han empleado alternativas de inertización por la vía de la ceramización y la vitrificación, que incluyen la fabricación de pigmentos, rellenos de materiales de construcción y cerámicos, así como la inclusión en esmaltes y vidrios (Gayo, 2017; J. Martínez y col., 2005; S. Martínez, 1996; Rincón, Hernández, y Romero, 2008). Ante la existencia de una planta productora de materiales de construcción aledaña a la empresa mecánica, esta investigación se propone como objetivo, evaluar la viabilidad técnica y ambiental de la disposición final del lodo galvánico en la fabricación de ladrillos refractarios.

\section{MATERIALES Y MÉTODOS Caracterización del lodo galvánico}

Primeramente, se realizó la caracterización del lodo galvánico generado por la empresa, para lo cual se tomaron muestras representativas semanales durante todo un año. Para lograr la representatividad, el muestreo se realizó según los requisitos de la norma cubana NC 671:06 Áridos. Toma de muestras (Oficina de Normalización, 2008). Las concentraciones de metales en el lodo fueron determinadas por espectrometría de absorción atómica en el laboratorio del departamento de análisis químico del Centro de Estudios Aplicados al Desarrollo Nuclear (CEADEN) de La Habana, Cuba, a una temperatura ambiente de $25 \stackrel{\circ}{\circ} \mathrm{C}$ y humedad relativa del $70 \%$.

Después de analizar las diferentes alternativas estudiadas mundialmente para el aislamiento de los lodos galvánicos, se seleccionó la de utilizar este residuo como soporte de la matriz cerámica del ladrillo refractario y de evaluar la misma. Esta decisión es tomada fundamentalmente dada la cercanía entre la fuente generadora del residuo y la fábrica de ladrillos refractarios de la empresa de materiales de construcción, donde se le daría un destino final al contaminante. Algunos estudios han revelado que esta alternativa es la más segura después de la disposición en el vidrio (Gayo, 2017). 
La evaluación de la alternativa propuesta se realizó desde los puntos de vistas técnico y ambiental. La evaluación ambiental requirió la medición de la presencia de $\mathrm{Cr}^{6+}$ en los gases generados durante la cocción del ladrillo que contiene una determinada proporción de lodo galvánico y posteriormente, a través de una prueba de lixiviación, se determinó la presencia del mismo en la solución de lixiviación que simula condiciones ambientales extremas a las que pudiera estar sometido el ladrillo durante su uso en un sistema constructivo. La evaluación técnica se basó en el cumplimiento de los requisitos de calidad del ladrillo refractario establecidos en el sistema de gestión de la empresa para la tecnología actual, añadiendo determinadas cantidades de lodo galvánico. Como variables respuestas para la evaluación técnica se seleccionaron los dos requisitos de calidad del ladrillo claves, la resistencia de la compresión y el porciento de absorción de agua. Esta evaluación técnica y ambiental se realizó sobre la base de un diseño experimental integrado que se presenta a continuación:

\section{Diseño experimental}

El diseño experimental comprende dos objetivos:

1. Evaluar la dependencia de la aparición de $\mathrm{Cr}^{6+}$ en los gases emitidos durante la cocción del ladrillo y el mantenimiento de los requisitos de calidad del mismo, con la temperatura del horno y la concentración de lodo galvánico.

2. Evaluar la dependencia de la aparición de $\mathrm{Cr}^{6+}$ en la solución de lixiviación que simula las condiciones de explotación del ladrillo, con la temperatura del horno y la concentración de lodo galvánico.

Para alcanzar estos objetivos, primeramente se evaluó la presencia de Cromo VI en los gases para tres niveles de temperatura, sin lodo galvánico y luego se realizó y ejecutó un diseño factorial a cinco niveles de los factores temperatura y concentración de lodo, auxiliándose para el diseño experimental del software estadístico statgraphics (StatPoint Technologies Inc., 2010). A continuación, se muestran los niveles de los factores:
Factores

Niveles

Temperatura $(\stackrel{\circ}{ } \mathrm{C}) 10501080111011401170$

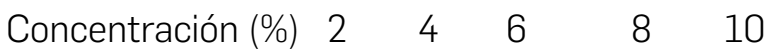

Para este diseño factorial, el número de experimento (N) se calculó como:

$\mathrm{N}=\mathrm{n}^{\mathrm{k}}=5^{2}=25$

Este experimento se desarrolló a nivel de laboratorio. Para la cocción de los ladrillos se utilizó una mufla de nacionalidad alemana marca Nabertherm, modelo LH 15/14, de una temperatura máxima de $1400{ }^{\circ} \mathrm{C}$ y un volumen de la cámara de 0,036 $\mathrm{m}^{3}$, que permitió la cocción de tres ladrillos por carga.

Para cada una de las combinaciones de factores y niveles se desarrollaron dos réplicas para un total de 75 muestras. Cada tratamiento se desarrolló en las siguientes condiciones:

Presión de compresión

Humedad de prensado 3,5 Mpa

Humedad de cocción

$8 \%$

Tiempo de cocción

$6 \%$

Temperatura del agua

para la disolución de Cromo $36{ }^{\circ} \mathrm{C}$

Se definieron las siguientes variables dependientes:

1. Concentración de $\mathrm{Cr}^{6+}$ en los gases de cocción.

2. Concentración de $\mathrm{Cr}^{6+}$ en la solución de lixiviación.

3. Resistencia a la compresión del ladrillo.

4. Absorción de agua del ladrillo.

La determinación de las dos primeras variables dependientes se corresponde con la evaluación ambiental. El método para la determinación de cromo VI en cada una de ellas se realizó según la norma cubana NC 93-01-121:88 (Oficina de Normalización, 1988).

La determinación de las dos últimas variables dependientes se corresponde con la evaluación técnica. Estas constituyen los principales requisitos de calidad del ladrillo asumidos por la empresa, según la norma cubana NC 54-303:85. Esta norma establece que la resistencia a la 
compresión del ladrillo debe ser superior a 200 $\mathrm{Kgf} / \mathrm{cm}^{2}$ y la absorción de agua debe estar entre 8 y $18 \%$.

\section{Determinación de la concentración de $\mathrm{Cr}^{6+}$ en los gases de cocción}

Teniendo en cuenta la alta solubilidad del Cromo VI en agua, los gases de la combustión de los ladrillos a la salida de la mufla se hicieron burbujear en un recipiente de cristal con un contenido de $2 \mathrm{~L}$ de agua destilada, para posteriormente realizarle el análisis cualitativo para la determinación del Cromo VI (NC 93-01121:88). Para lograr el burbujeo se empleó un ventilador centrífugo de $382 \mathrm{~m}^{3} / \mathrm{h}$, conectado a una manguera de $12,5 \mathrm{~mm}$ de diámetro interior, usada para la succión del ventilador procedente del horno mufla.

\section{Determinación de la concentración de $\mathrm{Cr}^{6+}$ en la solución de lixiviación}

Para realizar el ensayo de lixiviación se utilizó como referencia el método TCLP (Toxicity Characteristic Leaching Procedure por sus siglas en inglés) (SERAS, 2005). Este es el método de ensayo utilizado por la Agencia de Protección del Medio Ambiental en Estados Unidos. Según este método, para la preparación de las muestras debía procederse como sigue:

1. Se tritura la muestra a un tamaño de partícula de $5 \mathrm{~mm}$.

2. Se prepara una disolución de ácido acético a pH $=5$.

3. Se le agrega a la disolución acética la muestra del sólido con una relación de sólido - fluido de 19:1.

- Masa de la muestra - $25 \mathrm{~g}$.

- Volumen del fluido extractor - $475 \mathrm{ml}$. 4. Se realiza una mezcla con una agitación controlada.

- Tiempo de agitación - 16 h.

- Velocidad de agitación - 30 rpm.

- Temperatura - ambiente ( $25^{\circ} \mathrm{C}$ ).

5. Separación y filtración liquido / sólido.

- Filtro de 0,6 a 0,8 micras.

\section{Cálculo de la resistencia mecánica del ladrillo a la compresión}

Para la determinación de la resistencia mecánica a la compresión se utilizó una máquina para ensayo con una velocidad de aplicación de la carga de 0,196 kN/s (20 kgf/s). Se procedió situando la muestra sobre el plato de apoyo, haciendo coincidir el centro de la muestra con el centro del eje de la prensa, y se aumenta la presión lentamente hasta la destrucción total. La resistencia a la compresión $\mathrm{kgf} / \mathrm{cm}^{2}(\mathrm{RC})$ se determinó según la ecuación (1):

$\mathrm{R}_{\mathrm{C}}=\frac{\mathrm{F}}{\mathrm{S}}$

Donde:

$\mathrm{F}=$ carga destructiva $(\mathrm{kN})$

$\mathrm{S}=$ área de la superficie sometida a carga $\left(\mathrm{cm}^{2}\right)$

\section{Cálculo de la absorción de agua}

Para determinar la absorción de agua se pesaron inicialmente los ladrillos, lo que constituye su masa seca $\left(M_{1}\right)$. Luego se colocaron de canto en un recipiente y se cubrieron con agua hasta aproximadamente $5 \mathrm{~cm}$ por encima de estos. Se expusieron a estas condiciones durante 24 h y posteriormente se extrajeron y se secaron superficialmente con un paño. Seguidamente se pesaron determinándose su masa húmeda $\left(\mathrm{M}_{2}\right)$. La absorción de agua en \% (Abs) se determinó como aparece en la ecuación (2):

$$
\mathrm{Abs}=\frac{\left(\mathrm{M}_{2}-\mathrm{M}_{1}\right)}{\mathrm{M}_{1}} 100
$$

Para cada una de las variables respuestas que constituyen requisitos de calidad del producto (resistencia a la compresión y absorción de agua), se ajustaron modelos matemáticos que expresan la dependencia con las variables independientes temperatura de cocción y concentración de lodo. Para ambos casos el ajuste de los modelos se realizó empleando la herramienta Curve Fitting del software MATLAB (The MathWorks Inc., 2017) y también se realizaron, utilizando el software Statgraphics, análisis de regresión y de varianza. 


\section{RESULTADOS}

Del total de muestras de lodo examinadas durante el año de estudio para la caracterización, más del $90 \%$ poseían una concentración de cromo entre 20 y $25 \%$ peso y la de hierro oscilaba entre 5 y $8 \%$. En el resto de las muestras tomadas las concentraciones de estos metales eran inferiores a $20 \%$ para el cromo y a $5 \%$ para el hierro. Para el resto de los metales
Las concentraciones no rebasaron el $1 \%$ en ninguna de las muestras analizadas. En la Tabla 1 , se muestran los resultados promedios de los ensayos en cuanto al porciento peso de metales pesados sobre base seca para las muestras de más alta concentración de cromo y hierro, o sea, para el $90 \%$ de las muestras.

Tabla 1

Composición del lodo en \% peso de metales pesados

\begin{tabular}{cccccccccc}
\hline $\mathrm{Al}$ & $\mathrm{Si}$ & $\mathrm{Cr}$ & $\mathrm{Mn}$ & $\mathrm{Fe}$ & $\mathrm{Ni}$ & $\mathrm{Cu}$ & $\mathrm{Zn}$ & $\mathrm{Cd}$ & $\mathrm{Pb}$ \\
\hline \multirow{3}{*}{$<0.5$} & 0.22 & 22.6 & 0.51 & 6.1 & 0.36 & 0.42 & 0.76 & & 0.53 \\
& $+/-$ & $+/-$ & $+/-$ & $+/-$ & $+/-$ & $+/-$ & $+/-$ & $<0.02$ & $+/-$ \\
& 0.02 & 1.0 & 0.03 & 0.5 & 0.02 & 0.02 & 0.04 & & 0.03 \\
\hline
\end{tabular}

En la Tabla 2, se muestran los resultados de los ensayos de determinación de $\mathrm{Cr}^{6+}$ en los gases de cocción y en el lixiviado del ladrillo para las muestras procesadas según la tecnología actualmente establecida, sin lodo y en un intervalo de temperaturas entre 1060 y $1160 \stackrel{\circ}{ } \mathrm{C}$.

\section{Tabla 2}

Resultados de la determinación cualitativa de $\mathrm{Cr}^{6+}$ con la tecnología actual

\begin{tabular}{cccc}
\hline $\mathrm{N}^{\mathrm{o}}$ & $\begin{array}{c}\text { Temperatura de } \\
\text { cocción } \\
\left({ }^{\circ} \mathrm{C}\right)\end{array}$ & $\begin{array}{c}\text { Gases } \\
(\mathrm{si} / \mathrm{no})\end{array}$ & $\begin{array}{c}\text { Lixiviado } \\
(\mathrm{si} / \mathrm{no})\end{array}$ \\
\hline 1 & 1060 & No & No \\
2 & 1110 & No & No \\
3 & 1160 & No & No \\
\hline
\end{tabular}

En la Tabla 3, para la propia tecnología actual, se presentan los resultados de la determinación de la resistencia a la compresión y la absorción de agua.

Tabla 3

Resultados de la determinación de resistencia a la compresión y absorción de agua con la tecnología actual

\begin{tabular}{cccc}
\hline $\mathrm{N}^{\circ}$ & $\begin{array}{c}\text { Temperatura de } \\
\text { cocción } \\
\left({ }^{\circ} \mathrm{C}\right)\end{array}$ & $\begin{array}{c}\mathrm{R}_{\mathrm{C}} \\
\left(\mathrm{kgf} / \mathrm{cm}^{2}\right)\end{array}$ & $\begin{array}{c}\text { Abs } \\
(\%)\end{array}$ \\
\hline 1 & 1060 & 477 & 15 \\
2 & 1110 & 386 & 16 \\
3 & 1160 & 434 & 14 \\
\hline
\end{tabular}


Los resultados del desarrollo experimental se presentan en las Tablas 4 y 5. En la primera de ellas los resultados son cualitativos, relativos a la presencia o no de $\mathrm{Cr}^{6+}$ en los gases de cocción y en el lixiviado del ladrillo para las muestras procesadas a concentraciones de lodo de $2,4,6,8$ y $10 \%$ peso y a temperaturas entre 1050 y $1170 \stackrel{\circ}{ } \mathrm{C}$. En la Tabla 5, los resultados son cuantitativos y se corresponden con la resistencia a la compresión y la absorción de agua para las mismas concentraciones de lodo y temperaturas referidas anteriormente. Para cada caso, el valor reportado es el promedio del determinado para cada muestra con sus dos réplicas.

El mejor ajuste para el caso de la resistencia a la compresión se logró con un modelo polinómico bidimensional que alcanzó un coeficiente de determinación R2 de 0,9725 y cuya ecuación (3) se muestra a continuación:

$R_{C}=0,4933 T-21,64 C-114,6$

Donde, T es la temperatura de cocción $\left({ }^{\circ} \mathrm{C}\right)$ y $\mathrm{C}$ es la concentración de lodo (\%) en la masa de cocción.
En la Figura 1, se muestran las representaciones tridimensional y bidimensional del modelo obtenido para predecir el comportamiento de la resistencia a la compresión cuando cambian las dos variables independientes. En la sección superior de esa Figura 1 (representación tridimensional), se aprecia que la distribución de los puntos experimentales se aproxima al plano que representa el modelo obtenido, dando fe de la calidad del ajuste y el coeficiente de determinación logrado.

En la sección inferior de la Figura 1 (representación bidimensional), se puede apreciar que con el recuadro de línea discontinua de color rojo se ha limitado la zona de operación, la que quedó comprendida entre las temperaturas de cocción de 1060 y $1160 \stackrel{0}{ } \mathrm{C}$ y la concentración de lodo en la masa de cocción inferior a 7,3 \% aproximadamente. Esta es la zona en la que se pudiera operar el sistema para garantizar que la resistencia a la compresión se mantenga superior a $200 \mathrm{kgf} / \mathrm{cm}^{2}$ según lo establecido en la tecnología de producción del ladrillo. Los valores de RC se indican en la escala de colores que se representa en la columna de la derecha del gráfico. En el anexo se muestran los resultados de análisis de regresión y de varianza correspondientes a este modelo.

\section{Tabla 4}

Resultados de la determinación cualitativa de $\mathrm{Cr}^{6+}$ en gases y el lixiviado de los ladrillos para diferentes concentraciones de lodo y temperatura de cocción

\begin{tabular}{|c|c|c|c|c|c|c|c|c|c|c|c|}
\hline \multirow[b]{2}{*}{$\mathrm{N}^{\mathrm{o}}$} & \multirow{2}{*}{$\begin{array}{l}\text { Temperatura de } \\
\text { cocción } \\
\left({ }^{\circ} \mathrm{C}\right)\end{array}$} & \multicolumn{2}{|c|}{$2 \%$ de lodo } & \multicolumn{2}{|c|}{$4 \%$ de lodo } & \multicolumn{2}{|c|}{$6 \%$ de lodo } & \multicolumn{2}{|c|}{$8 \%$ de lodo } & \multicolumn{2}{|c|}{$10 \%$ de lodo } \\
\hline & & $\begin{array}{l}\text { Gases } \\
\text { (si/no) }\end{array}$ & $\begin{array}{l}\text { Lixiviado } \\
\text { (si/no) }\end{array}$ & $\begin{array}{l}\text { Gases } \\
\text { (si/no) }\end{array}$ & $\begin{array}{l}\text { Lixiviado } \\
\text { (si/no) }\end{array}$ & $\begin{array}{l}\text { Gases } \\
(\mathrm{si} / \mathrm{no})\end{array}$ & $\begin{array}{l}\text { Lixiviado } \\
\text { (si/no) }\end{array}$ & $\begin{array}{l}\text { Gases } \\
(\mathrm{si} / \mathrm{no})\end{array}$ & $\begin{array}{c}\text { Lixiviado } \\
\text { (si/no) }\end{array}$ & $\begin{array}{l}\text { Gases } \\
(\mathrm{si} / \mathrm{no})\end{array}$ & $\begin{array}{c}\text { Lixiviado } \\
\text { (si/no) }\end{array}$ \\
\hline 1 & 1050 & No & No & No & No & No & No & No & No & No & $\mathrm{Si}$ \\
\hline 2 & 1080 & No & No & No & No & No & No & No & $\mathrm{Si}$ & No & $\mathrm{Si}$ \\
\hline 3 & 1110 & No & No & No & No & No & No & No & $\mathrm{Si}$ & No & No \\
\hline 4 & 1140 & No & No & No & No & No & No & No & No & No & $\mathrm{Si}$ \\
\hline 5 & 1170 & No & No & No & No & No & $\mathrm{Si}$ & No & $\mathrm{Si}$ & No & $\mathrm{Si}$ \\
\hline
\end{tabular}

\section{Tabla 5}

Resultados de la determinación de resistencia a la compresión y absorción de agua para diferentes concentraciones de lodo y temperatura de cocción

\begin{tabular}{|c|c|c|c|c|c|c|c|c|c|c|c|}
\hline \multirow[b]{2}{*}{$\mathrm{N}^{\mathrm{o}}$} & \multirow{2}{*}{$\begin{array}{l}\text { Temperatura de } \\
\text { cocción } \\
\left({ }^{\circ} \mathrm{C}\right)\end{array}$} & \multicolumn{2}{|c|}{$2 \%$ de lodo } & \multicolumn{2}{|c|}{$4 \%$ de lodo } & \multicolumn{2}{|c|}{$6 \%$ de lodo } & \multicolumn{2}{|c|}{$8 \%$ de lodo } & \multicolumn{2}{|c|}{$10 \%$ de lodo } \\
\hline & & $\begin{array}{c}\mathrm{R}_{\mathrm{C}} \\
\left(\mathrm{Kgf} / \mathrm{cm}^{2}\right)\end{array}$ & $\begin{array}{l}\text { Abs } \\
(\%)\end{array}$ & $\begin{array}{c}\mathrm{R}_{\mathrm{C}} \\
\left(\mathrm{Kgf} / \mathrm{cm}^{2}\right)\end{array}$ & $\begin{array}{l}\text { Abs } \\
(\%)\end{array}$ & $\begin{array}{c}\mathrm{R}_{\mathrm{C}} \\
\left(\mathrm{Kgf} / \mathrm{cm}^{2}\right)\end{array}$ & $\begin{array}{l}\text { Abs } \\
(\%)\end{array}$ & $\begin{array}{c}\mathrm{R}_{\mathrm{C}} \\
\left(\mathrm{Kgf} / \mathrm{cm}^{2}\right)\end{array}$ & $\begin{array}{l}\text { Abs } \\
(\%)\end{array}$ & $\begin{array}{c}\mathrm{R}_{\mathrm{C}} \\
\left(\mathrm{Kgf} / \mathrm{cm}^{2}\right)\end{array}$ & $\begin{array}{l}\text { Abs } \\
(\%)\end{array}$ \\
\hline 1 & 1050 & 359 & 16 & 325 & 16 & 287 & 16 & 227 & 17 & 183 & 18 \\
\hline 2 & 1080 & 364 & 15 & 331 & 15 & 306 & 17 & 229 & 16 & 200 & 19 \\
\hline 3 & 1110 & 368 & 16 & 343 & 16 & 314 & 16 & 260 & 14 & 210 & 17 \\
\hline 4 & 1140 & 410 & 19 & 364 & 19 & 322 & 19 & 299 & 19 & 219 & 19 \\
\hline 5 & 1170 & 424 & 15 & 366 & 15 & 328 & 17 & 306 & 19 & 235 & 18 \\
\hline
\end{tabular}




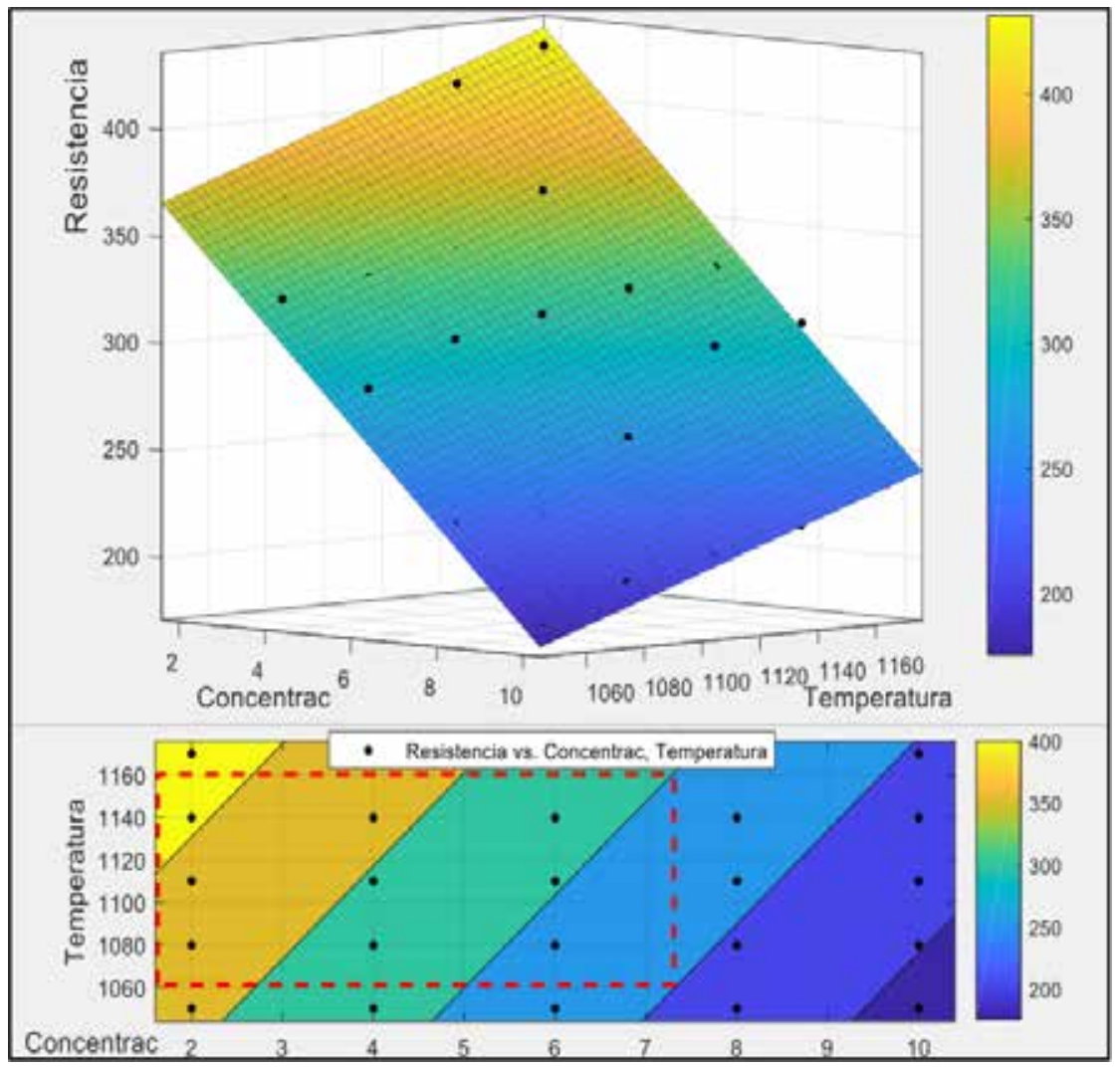

Figura 1. Representaciones tridimensional y bidimensional del modelo obtenido para predecir el comportamiento de $\mathrm{R}_{\mathrm{C}}$

En el caso de la absorción de agua no se logró un modelo con buen ajuste y el coeficiente de determinación $\mathrm{R}^{2}$ alcanzado fue de solamente de 0,259. En el anexo, también se muestran los resultados del análisis de regresión y de varianza correspondientes a este ajuste de la variable absorción de agua.

Estos resultados declaran a la temperatura como una variable poco significativa, por lo que la variación de esta debe tener poca importancia en el comportamiento de la absorción de agua y debe ser eliminada del modelo. No obstante, en la Figura 2 que se presenta a continuación, se aprecia que los valores promedios de absorción de agua siguen una tendencia al incremento con el aumento de la concentración de lodo en la composición del ladrillo. La Abs promedio se obtienen a partir de todos los valores de Abs para cada concentración independientemente de la temperatura de cocción. 


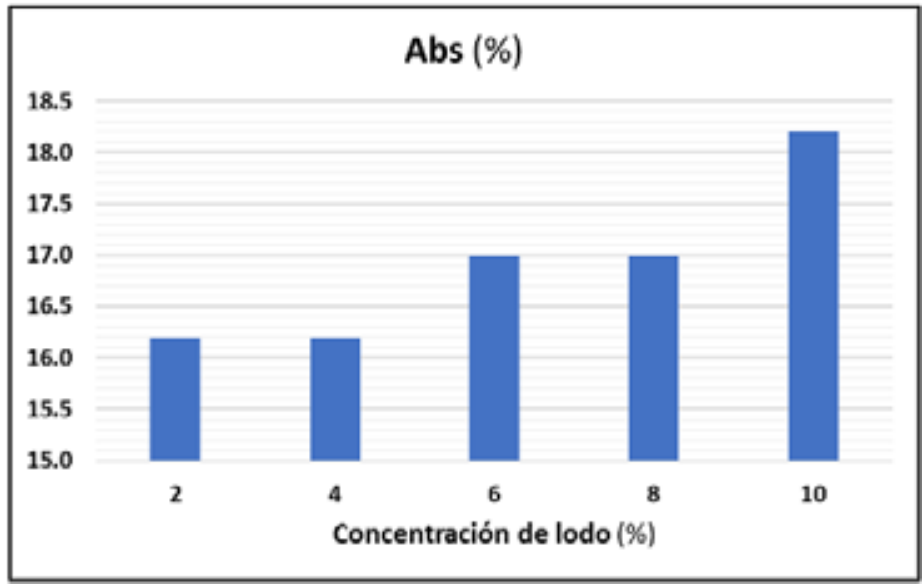

Figura 2. Comportamiento de la Abs promedio con el incremento de la concentración de lodo

\section{DISCUSIÓN}

\section{Evaluación de las emisiones gaseosas.}

El análisis cualitativo para determinar la presencia de $\mathrm{Cr}^{6+}$ practicado al agua destilada, donde se hicieron burbujear los gases originados por la cocción de los ladrillos, no reveló presencia de dicho ión para ninguna de las cinco concentraciones de lodo galvánico evaluadas. A pesar de la alta sensibilidad del método de determinación de la norma cubana NC 93-01121:88, usada en el ensayo, el $\mathrm{Cr}^{6+}$ no es detectado en la solución, pero en cualquier caso, se hace necesario cuantificar su contenido en los gases utilizando un método certificado y compararlo con el establecido en la norma cubana NC 9302-202: 97 (Oficina de Normalización, 1997) y su enmienda obligatoria NC 39: 99 Calidad del aire. Requisitos higiénico sanitarios (Oficina de Normalización, 1999), que para este caso es de $0,0015 \mathrm{mg} / \mathrm{m}^{3}$.

\section{Evaluación técnica y ambiental del ladrillo.}

En la medida en que aumenta el porciento en peso del lodo galvánico en la masa de cocción del ladrillo se observa una disminución de la resistencia a la compresión y un aumento de la absorción de agua, de tal modo que para los ladrillos fabricados con el $10 \%$ en peso de lodo, estos requisitos de calidad adquirían valores fuera de los límites de aceptación o muy cercanos a este, que para el caso de la resistencia a la compresión no debe ser menor de 200 Mpa y para la absorción de agua no mayor de 18 \%. Para los ladrillos con el 10 $\%$ peso de lodo galvánico no se garantiza el cumplimiento de las especificaciones de calidad establecido en la norma cubana NC 54-303:85 (Oficina de Normalización, 1985) que se utiliza como referencia para el control de la calidad del ladrillo. Para el caso de los ladrillos con el 2, 4, 6 y $8 \%$ peso de lodo galvánico, se cumple con la viabilidad técnica especificada en la norma para cualquiera de las temperaturas evaluadas entre 1050 y $1170 \stackrel{\circ}{ } \mathrm{C}$.

Un análisis de regresión múltiple realizado utilizando la aplicación estadística Statgraphics, permitió determinar que existe una relación estadísticamente significativa entre los factores temperatura y concentración de lodo con la resistencia a la compresión. Igualmente se ajustó un modelo polinómico entre las referidas variables que permitirá determinar el valor de la resistencia a la compresión para diferentes condiciones de operación.

Para el caso de la relación entre las variables independientes y la variable dependiente absorción de agua, luego de aplicar el Statgraphics en un análisis de regresión múltiple, se recomienda eliminar la temperatura del modelo. Estos resultados indica que la temperatura no es un factor esencial en el control de la absorción de agua del ladrillo y seguramente existen otros factores influyentes no considerados en este experimento. De cualquier manera, los resultados del análisis de regresión que se muestra en el anexo y la observación de la Figura 2, corroboran 
que la relación de la concentración de lodo y la absorción de agua guardan una relación estadísticamente significativa.

La zona marcada con línea discontinua de color rojo en la Figura 1, indica que durante la cocción del ladrillo se puede operar a una temperatura entre 1060 y $1160 \stackrel{\circ}{C}$ (intervalo de temperatura es el establecido en la tecnología actual) y a concentraciones másicas de lodo galvánico por debajo de $7,3 \%$. En estas condiciones se garantizarán los requisitos de calidad del ladrillo. Como resultado de la evaluación ambiental del ladrillo durante la explotación, en la Tabla 4 se observa que para bajas concentraciones de lodo de 2 y $4 \%$ no se detecta presencia de cromo en estado hexavalente en la solución del lixiviado. A concentraciones mayores se detecta la presencia del ion y cada vez en mayor número de muestras.

Se considera que en el caso del $10 \%$ de lodo, el hecho de no detectar el $\mathrm{Cr}^{6+}$ en uno de los niveles de temperatura puede haber sido resultado de un error en la determinación o en la aplicación del método de lixiviación, sin embargo, está clara la tendencia de aparición de $\mathrm{Cr}^{6+}$ con el aumento de la concentración másica de lodo en la mezcla utilizada como materia prima en la fabricación del ladrillo. La inexistencia del mismo en la solución de lixiviación para concentraciones de lodo hasta el $4 \%$ avala la viabilidad ambiental del ladrillo para las condiciones de explotación y con el método de disposición bajo estudio. Se debe significar que el método de ensayo TCLP de la prueba de lixiviación es muy exigente y mide la conversión del cromo trivalente en condiciones extremas que probablemente nunca se asemejarán a las condiciones de explotación de la gran mayoría de los ladrillos fabricados.

Los resultados anteriores revelan la viabilidad técnica y ambiental de realizar la disposición final de lodo galvánico como material de relleno en la fabricación de ladrillos. Aunque 4 $\%$ de lodo en la masa de cocción parezca una proporción pequeña, esta es suficiente para disponer de forma adecuada de todo el volumen de lodo generado por la empresa mecánica, cuyo volumen de emisiones anuales de este lodo es muy inferior al $4 \%$ de la masa de cocción que demanda la producción de ladrillos en la planta productora.

Aunque esta alternativa de disposición es viable, se ha seleccionado teniendo en cuenta fundamentalmente la cercanía entre el emisor y la fábrica de ladrillos. Pudieran explorarse otras alternativas basadas en la vitrificación u otras formas de disposición reportadas en la literatura (Gayo, 2017; J. Martinez et al., 2005; S. Martinez, 1996; Rincon et al., 2008). Es importante también, monitorear los posibles cambios que pueden ocurrir en la composición de lodo emitido por la empresa mecánica ante modificaciones tecnológicas o de capacidades. Cambios de esa naturaleza podrían modificar radicalmente la visión sobre los resultados que se muestran en esta investigación.

\section{CONCLUSIONES}

Esta investigación demostró que es viable técnica y ambientalmente la disposición final de lodo galvánico en la mezcla para la fabricación de ladrillos refractarios. Los ladrillos fabricados con una concentración de lodo de hasta el 4 $\%$ peso, en el rango de temperaturas de la tecnología actual, cumplen con los requisitos de calidad exigidos por la norma de referencia del sistema de gestión de la empresa. El modelo matemático obtenido demostró la capacidad para determinar la resistencia a la compresión en función de la concentración de lodo y la temperatura de cocción. Con esta concentración de hasta el $4 \%$ y para cualquier temperatura de cocción se garantiza que el ladrillo sea seguro durante su explotación, sin riesgos de que su contenido de cromo trivalente se pueda convertir a $\mathrm{Cr}^{6+}$. También se logra que durante la cocción de los ladrillos no se generen gases con concentraciones detectables de $\mathrm{Cr}^{6+}$, elemento potencialmente más nocivo para la salud humana entre los posibles constituyentes del lodo galvánico. 


\section{REFERENCIAS BIBLIOGRÁFICAS}

Agencia Presidencial de Cooperación. (2017). A-OT-013 Manual de gestión de los residuos o desechos peligrosos. In (pp. 1-28). Bogotá, Colombia. Disponible en: https://www.apccolombia.gov.co/sites/ default/files/archivos_usuario/2016/a-ot013manualgestionresiduosdesechospeligr ososv3.pdf

Andreola, F., Barbieri, L., Cannio, M., Lancellotti, L., Siligardi, C., y Soragni, E. (2006). Physical-chemical characterization of a galvanic sludge and its inertization by vitrification using container glass. Waste Management and the Environment III, 92, 23-30. Retrieved from doi:http://dx.doi. org/10.2495/WM060031

Becerra, B., Soria, C., Jaramillo, F., y Moreno,J. L. (2014). Trastornos a la salud inducidos por cromo y el uso de antioxidantes en su prevención o tratamiento. Journal of Pharmacy \& Pharmacognosy Research, 2(2), 19-30. Disponible en: https://www. redalyc.org/pdf/4960/496050267001.pdf

CITMA. (1999). Resolucion № 87. Regulaciones para el ejercicio de las funciones de autoridad nacional y punto de contacto del convenio de basilea sobre el control de los movimientos transfronterizos de desechos peligrosos y su eliminación y otras disposiciones para la gestión ambientalmente racional de estos desechos. Habana, Cuba.

Gayo, G. X. (2017). Fabricación de pigmentos cerámicos con criterios sustentables. (Tesis de doctorado), Universidad Jaume, Castellón de la Plana, España. DOI: http:// dx.doi.org/10.6035/14107.2017.665463

Martínez, J., Mallo, M., Lucas, R., Álvarez, J., Salvarrey, A., y Gristo, P. (2005). Guía para la gestión integral de residuos peligrosos. Fundamentos. Tomo I. In (pp. 164).

Martínez, S. (1996). Reciclaje y tratamiento de residuos. Acta Geológica Hispánica, 30(13), 63-72. Disponible en: https://raco.cat/ index.php/ActaGeologica/issue/view/6392
Oficina de Normalización. (1985). NC 54-303:85 Materiales y productos de la construcción. Refractarios de chamota y semiacidos de uso común. Especificaciones de calidad. In (pp. 10). Habana,Cuba.

Oficina de Normalización. (1988). NC 93-01121:88 Sistema de normas para la proteccion del medio ambiente. Medio ambiente. Hidrosfera. Determinacion de cromo hexavalente. In. Habana, Cuba.

Oficina de Normalización. (1997). NC 9302-202:97 Atmósfera. Requisitos higiénico sanitarios. Concentraciones máximas admisibles, alturas mínimas de expulsión y zonas de protección sanitarias. In (Vol. , pp. 10). Habana,Cuba.

Oficina de Normalización. (1999). NC 39:99 Calidad del aire. Requisitos higienicosanitarios. In (pp. 89). Habana, Cuba.

Oficina de Normalización. (2008). NC 671:08 Aridos. Toma de muestras. In (pp. 12). Habana,Cuba.

Organisation for Economic Cooperation and Development (OECD). (2009). Guidance manual for the control of transboundary movements of recoverable wastes. Paris, France.

Presidencia de la Republica. (2005). Decreto número (4741). Bogotá, Colombia.

Rincón, J. M., Hernández, M., y Romero, M. (2008). Vitrificacion de residuos industriales inorganicos para la produccion de nuevas fritas, asi como de plaquetas porcelanicas $y$ vitroceramicas. Paper presented at the I Simposio Iberoamericano de Ingeniería de Residuos. Disponible en: http://www.redisa. net/doc/artSim2008/tratamiento/A23.pdf

Rodríguez, D. (2017). Intoxicación ocupacional por metales pesados. MEDISAN, 21(12), s.p.

Scottish Environment Protection Agency (SEPA). (2021). Guidance on the 
classification and assessment of waste. Technical Guidance WM3. UK.

SERAS. (2005). Toxicity characteristic leaching procedure (TCLP). In Standard Operating Procedures (Vol. SOP 1831, pp. 1-12). United States of America: United States Environmental Protection Agency. Disponible en: https://clu-in.org/download/ ert/1831-r00.pdf

Somoza Cabrera, J. (2009). Evaluación del medio ambiente cubano. In A. Fernández y R. Pérez (Eds.), Contexto político, económico, ambiental y social cubano (pp. 11-49). Habana, Cuba: Instituto Nacional de Investigaciones Económicas.

StatPoint Technologies Inc. (2010). Statgraphics Centurion XVI (Version 16.2.04) [Programa de computador]. Warrenton, VA, USA: StatPoint Technologies Inc.

The MathWorks Inc. (2017). Matlab (Version 9.3.0.713579 (R2017b)) [Programa de computador]. Natick, MA, USA: The MathWorks Inc.

Tirado, L. R., González, F. D., Martínez, L. J., Wilches, L. A., y Celedón, J. N. (2015). Niveles de metales pesados en muestras biológicas y su importancia en salud. Revista Nacional de Odontología., 11(21), 83-99. DOl:http://dx.doi.org/10.16925/ od.v11i21.895

UNEP. (2000). Methodological guide for the undertaking of national inventories of hazardous wastes within the framework of the basel convention. Ginebra, Suiza. 